\title{
ROLES FOR AUTONOMOUS PHYSIOLOGIC AGENTS; AN OXYGEN SUPPLY AND DEMAND EXAMPLE
}

\author{
Meyer Katzper \\ 2 Locks Pond Court \\ SIA \\ Rockville, MD 20854, U.S.A.
}

\begin{abstract}
In the study of physiologic systems control, lumped parameter and differential equation techniques are standard approaches. Application of these techniques to the study of oxygen supply to tissues is discussed. It is then proposed that progress in dealing with heterogeneous physiologic systems is likely to proceed from the techniques of agent based modeling in the form of autonomous physiologic agents.
\end{abstract}

\section{INTRODUCTION}

The human being can be considered to be composed of many dependent but autonomous components which act in concert. Each individual cell contains a complete blueprint to guide it. At the same time each cell is dependent on other cells for its entire existence. Thus, to carry out their functions our cells must be supplied with oxygen and nutrients. There have been many studies, both experimental and theoretical investigating these processes. Sophisticated modeling and simulation has been carried out. However, these studies have not taken into account the autonomous aspect of the cellular entities. Of course the cell is dependent on its environment for all of its nutrients and subject to many controlling factors. At the same time the cell has significant independent control over its development, what it ingests and what it emits to the surrounding milieu.

This paper will deal with the themes of lumped parameter and control representation versus control that emerges in the context of an independent agent representation. The main example will be oxygen transport and delivery from capillaries to cells. Our calculations and findings depend on the tool we use. The goal here is to elucidate the place of the different representations in understanding and calculating physiological phenomena. The point is made that the agent based approach is currently underused and underappreciated.

\section{BACKGROUND}

The human body has a constant need for a sufficient oxygen supply. For this reason many research efforts have focused on oxygen supply to tissues. The circulatory system is the vehicle for this function. The proximate effectors of oxygen supply are the capillary networks.

The Krogh cylinder is a concept introduced by August Krogh (Krogh 1919). Krogh was the 1920 Nobel Laureate in Medicine for his discovery of the capillary motor regulating mechanism. Krogh postulated a cylindrical unit structure as the volume to be used for calculation of oxygen transport. There are multiple parallel cylindrical structures assumed to be supplying a larger volume of tissue. This idealization implies that each section of capillary is responsible for the supply to a corresponding cylindrical section of surrounding tissue.

Real capillaries and tissue are far more complex in their geometry. This idealized model is amenable to an analytic solution. Note that this idealization makes fairly strong assumptions. The tissue surrounding a capillary is actually a heterogeneous composite of materials with many metabolic reactions taking place.

In considering only oxygen supply and utilization the idealized assumption is that the chemical reactions are continuously, evenly distributed. The analytic solution for a single cylinder is sufficient as symmetry about the axis of capillary-tissue cylinder is assumed. As a consequence, there is a cylindrical surface about a capillary across which transport from surrounding capillaries just balances transport toward these capillaries. Therefore, net transport rates vanish along this surface, which can be called a "no-flux" surface and so the solution for the unit cylinder is the complete solution. With this construct, under conditions approximating anoxia Krogh's solution defined a "lethal corner" where the lack of oxygen was first felt.

It is worth noting that Newton invented the calculus to deal with problems of a space that was uniform and homogeneous. The objects of study in such a space were the sun and its planets. Similarly, trajectories of objects on earth 
moved in a homogeneous space. For such problems differential equations, modeling objects advancing through infinitesimal indistinguishable intervals, were an ideal representation which matched reality. The laws formulated using differential equations had broad general validity. Clearly, in attempting to model physiological situations with differential equations the representation matches reality only crudely. Using a discrete modeling basis, such as an agents and patches framework we should be able to capture physiological situations more realistically. This capability is now available to us due to computer calculational power. However, in modeling the in-homogeneity and variability we observe we are in danger of loosing the generality of our findings. Generality is not to be found in the specific solutions but in the agent behavioral rules which generate observable like behavior.

I here show different approaches to the area of oxygen supply to tissues to demonstrate the different views possible for a single situation. First I will demonstrate a control approach to blood flow using difference equations implemented in Stella. Next I will discuss modifications of the basic Krogh model for advanced applications. Finally I will show an agent based approach to oxygen transport in capillaries, implemented in NetLogo.

\section{OXYGEN LEVEL CONTROL}

It has clearly been established that that there is local control of blood flow in response to tissue need (Guyton and Hall 2005). Under a wide range of conditions the body can avoid anoxia. As the demand for oxygen increases signals are sent and received by the capillary system. As a result closed capillaries are opened. In the long term, if there is a persistent lack of sufficient oxygen, new capillaries develop to supply the deficient area.

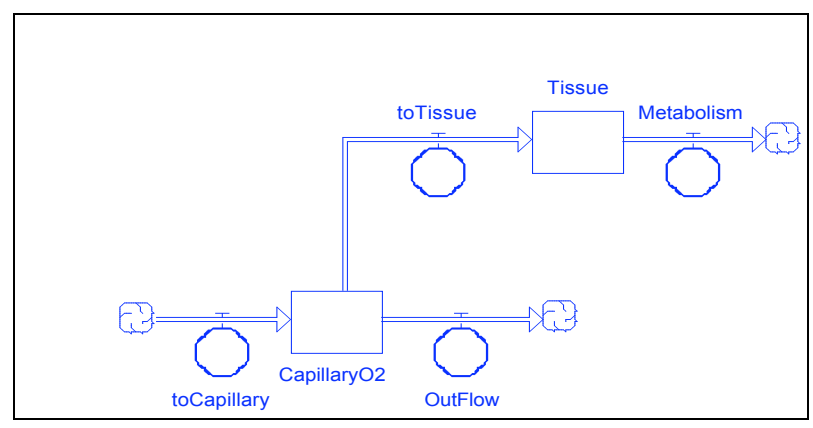

Figure 1:Model of oxygen supply from capillary to tissue

A representation of the essence of this process is shown in Figure 1 using Stella graphical notation. The model is not a diffusion model. It shows what can be expected compartmentally under specified supply, demand and control conditions. We assume a time and materials dependent tissue process. We assume a stand alone supply source. We provide a valve to tap different fractions of the supply source as needed. Feedback control is built into the determination of the fraction so as to meet metabolic needs. Feedback control from the tissue to the capillary is also built in to modify capillary oxygen supply as needed. Results are displayed in Figure 2 for a sample instance. In the instance displayed there has been a precipitous drop in tissue oxygen. The feedback mechanism quickly extracts a large amount of oxygen from the capillary and reequilibrates. The capillary gets back to its original level more slowly. In general additional demand for oxygen can be met by extracting a larger fraction of available oxygen from the capillary (Law 1999). When more oxygen is required additional capillaries will open up.

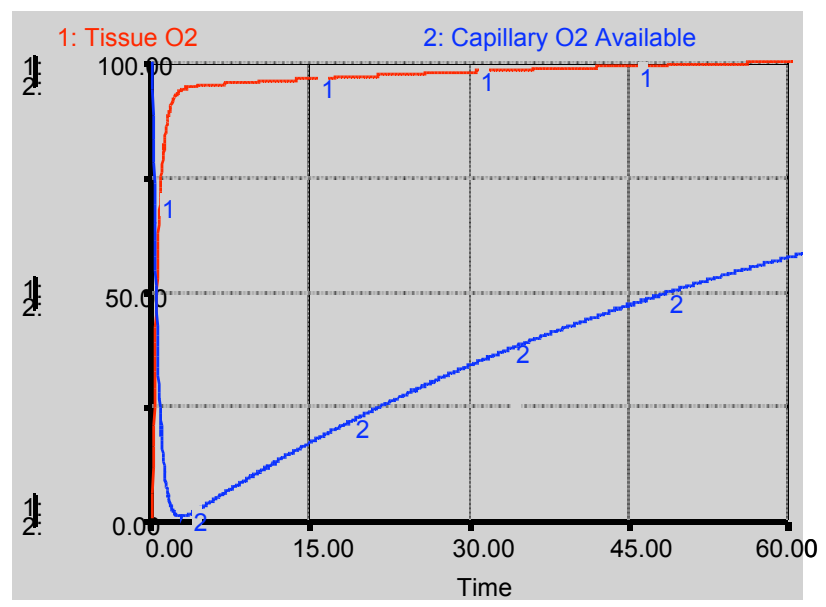

Figure 2: Sample simulation results

This level of lumped parameter modeling is sufficient to study the effects of various control mechanisms. To study oxygen distribution along the length of the capillary we turn to the Krogh cylinder.

\section{KROGH CYLINDER APPLICATIONS}

Modifications and enhancements can be made in the Krogh cylinder model to address other questions and to refine the model to incorporate additional vital factors.

One area which entails much which is unknown is consciousness and sleep. It was surmised by Katzper and Brown that an aspect of the different mental patterns during sleep and wakefulness involved different oxygen distribution and utilization. An inner and outer Krogh cylinder model was constructed with different diffusivity in the two regions. This model was analytically solved and simulated for various diffusion parameters.(Brown and Katzper 1970). It was shown that such a construct would allow for differing oxygen levels in the two regions and presumably different nerve firing patterns. 
There has been an enormous amount of experimental and theoretical work over the years relating to oxygen transport to tissues. A comprehensive review with a focus on the Krogh cylinder is by Popel (Popel 1989). Bassingthwaighte has been involved in many advanced modeling studies as can be seen by doing a Google search on his name and oxygen. Recent work is the basis for a web model (Dash and Bassingthwaighte 2006). Here I quote a few descriptive lines of recent work from the web site (Bassingthwaighte 2007) "This model describes the exchange of $\mathrm{O} 2, \mathrm{CO} 2, \mathrm{HCO} 3$ - and $\mathrm{H}+$ between the $\mathrm{RBCs}$ (red blood cells) and plasma along the arterial vasculature. Both $\mathrm{O} 2$ and $\mathrm{CO} 2$ exist in soluble and bound to hemoglobin $(\mathrm{Hb})$ states in the $\mathrm{RBC}$ while there is only a soluble state in the plasma. Disequilibrium between the partial pressure of $\mathrm{O} 2$ and $\mathrm{CO} 2$ in plasma and $\mathrm{RBCs}$ can exist at the entry to the arterial vasculature." This partial quote is presented to provide the reader with an idea as to the level of detail that has been developed in this area of study. With all this level of detail in the differential equation approach to blood flow the active participation of tissues in affecting capillaries and their blood flow and thus the oxygen reaching the tissues is not directly incorporated in the equations.

\section{PHYSIOLOGIC AGENT BASED MODELS}

There are many platforms for carrying out agent based programming. NetLogo is the one used here (Wilensky 1999). NetLogo is a multi-agent programming language and modeling environment for simulation. It is suitable for modeling complex systems evolving over time. The world consists of a grid of patches which can independently hold variables and thus are suitable for representing cellular entities. The active agents are called turtles and they roam the world of patches and have their own properties. Modelers can give instructions to many independent turtle "agents" all operating concurrently. This makes it possible to explore connections between our postulated micro-level behaviors of these agents and observed macro-level phenomena. Such correspondence is labeled emergent behavior and depends on the turtles and patches interactions. This platform provides a good way to simulate distributed systems.

The diffusion of oxygen from capillary blood flow to tissues is modeled. The oxygen molecules are flowing down the capillary and diffusing into the surrounding tissue. They are mostly attached to the hemoglobin of the red blood cells which are not shown.

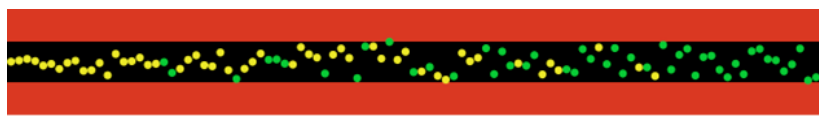

Figure 3: Oxygen and carbon dioxide flow along the capillary
After metabolism the resulting carbon dioxide is removed. A NetLogo representation of this process is shown in Figure 3

The red lines are the capillary boundaries and surrounding tissue. Particles which exit the channel and are metabolized are marked by a change in color (from yellow to green in this implementation). This symbolizes an exchange of $\mathrm{O} 2$ to $\mathrm{CO} 2$.

We can obtain quantitative measures of the fate of the oxygen and carbon dioxide as they traverse the capillary by means of a histogram. These distributions are not stationary. They are time varying due to stochastic factors but also due to varying deterministic local demand. Through following the varying demand an improved characterization of the process will be possible. In Figure 4 and Figure 5 histogram snapshots of a low diffusion case and a high diffusion case are shown as the capillary is traversed. Even though these patterns will be seen to fluctuate with time the high and low diffusion cases can easily be distinguished.

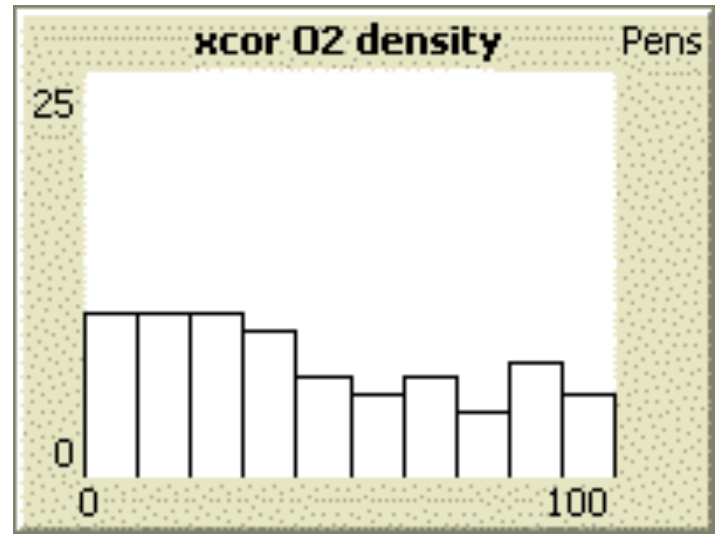

Figure 4: Oxygen distribution along the capillary for the low diffusion case

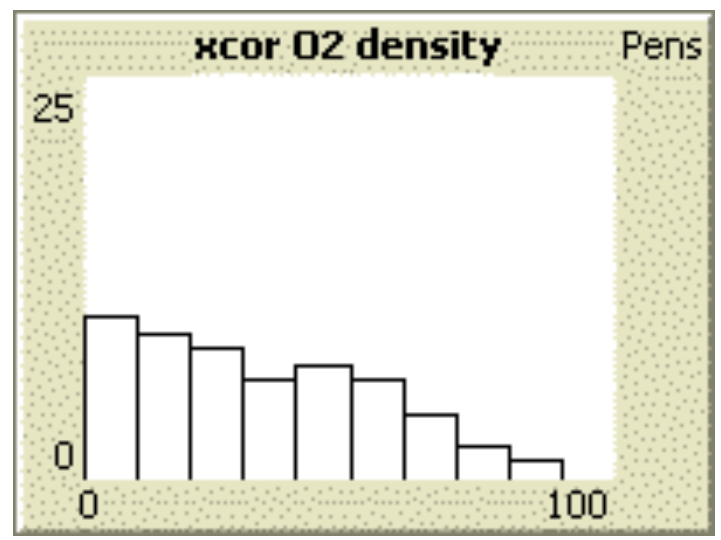

Figure5: Oxygen distribution along the capillary for the high diffusion case 
We can obtain a picture of the interaction of the oxygen agents with the cell patches as they dynamically interact. Our scenario starts with an anoxic segment of tissue surrounding the capillary channel. Capillary flow with oxygen is restarted. The anoxic cells are colored. The cells whose oxygen needs are met are white. Oxygen molecules are shown in yellow, carbon dioxide molecules are shown in green. The Figure 6 snapshot is taken after flow has proceeded for a time but has not achieved an equilibrium state for the oxygen supply to tissue. The pattern we see is consistent with the results for the Krogh cylinder. We see why there would be a lethal corner in the rightmost edge of the capillary channels predicted by Krogh. Here we can visualize the lethal corner; an area where $\mathrm{O} 2$ cannot reach and $\mathrm{CO} 2$ builds up on the Krogh cylinder. This can occur in each capillary bed. It can be small and reversible, or large and irreversible. It is important to avoid such a situation in circumstances such as operations.

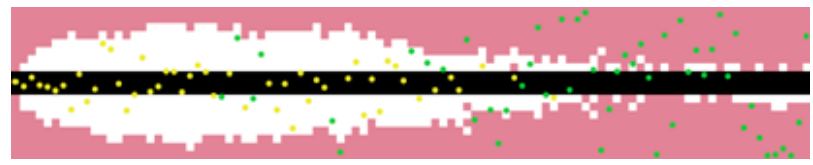

Figure 6: Oxygen supply to tissue

\section{CONCLUSION}

In view of the fact that differential and difference equations have been the preferred research tools for many years there are many more studies using these tools than those using agent based formulations for physiologic studies. Many advances have been made using these approaches. However, the intrinsic inhomogeneity of physiologic systems is not captured by these differential equation approaches. The agent based platform, an example of which we have shown here, should allow future advances with a strong correspondence to physiologic reality.

\section{DISCLAIMER}

The opinions expressed in this paper are the professional views of the author and do not necessarily reflect the official position of the U.S. Food and Drug Administration.

\section{REFERENCES}

Bassingthwaighte 2007. Available via <http://www . physiome.org/model/doku.php?do=show \& id=Transport_Physiology3ACombined_c onvetion dision permeation reaction 3AArtrial flow with $02 \mathrm{CO}^{-}{ }^{-} \mathrm{HCO} 3$ and $\mathrm{H}$ exchange ${ }^{\circ}$ Amodel detail> [Accessed June 5, 2007]
Brown, B., and M. Katzper. 1970. Brain Oxygen Supply and Electrical Activity. Neuroelectric Research, ed. D.V. Reynolds and A. E. Sjoberg, Chapter 40. Springfield: C. C. Thomas.

Dash, R. K. and J. B. Bassingthwaighte. 2006 Simultaneous blood-tissue exchange of oxygen, carbon dioxide, bicarbonate and hydrogen ion. Annals of Biomedical Engineering 34:1129-1148.

Guyton, A. and J. E. Hall. 2005. Textbook of Medical Physiology.11th ed. Amsterdam: Elsevier.

Krogh, A. 1919. The Number and Distribution of Capillaries in Muscle With Calculations of the Oxygen Pressure Head Necessary for Supplying the Tissue. Journal of Physiology (London) 52: 391-409.

Law, R. 1999. The Physiology of Oxygen Delivery Physiology Issue 10 Article 3. Available via <http://www.nda.ox.ac.uk/wfsa/html/u 10/u1003_01.htm > [accessed June 5, 2007].

Popel A. S. 1989. Theory of oxygen transport to tissue. Critical Review of Biomedical Engineering. 17(3):257-321.

Wilensky U. 1999. NetLogo. Available via <http:// ccl.northwestern.edu/netlogo> [accessed June 5, 2007].

\section{AUTHOR BIOGRAPHY}

MEYER KATZPER is a reviewer, analyst and modeler for the Food and Drug Administration. He has carried out modeling, simulation and data analysis to enhance the drug review process. His web site is <http://mysite . verizon.net/vze70xvs/shs/shsmain.htm> 